\title{
Differential approximation of MIN SAT, MAX SAT and related problems
}

\author{
Bruno Escoffier Vangelis Th. Paschos \\ LAMSADE, Université Paris-Dauphine \\ Place du Maréchal De Lattre de Tassigny, 75775 Paris Cedex 16, France \\ \{escoffier, paschos\}olamsade.dauphine.fr
}

22nd June 2004

\begin{abstract}
We present differential approximation results (both positive and negative) for optimal satisfiability, optimal constraint satisfaction, and some of the most popular restrictive versions of them. As an important corollary, we exhibit an interesting structural difference between the landscapes of approximability classes in standard and differential paradigms.
\end{abstract}

\section{Introduction and preliminaries}

In this paper we deal with the approximation of some of the most famous and classical problems in the domain of the polynomial time approximation theory, the MIN and MAX SAT as well as the MIN and MAX DNF and some of their restricted versions, namely MAX and MIN $k$ and EkSAT and MAX and MIN $k$ and E $k$ DNF. We study their approximability using the so-called differential approximation ratio which, informally, for an instance $x$ of a combinatorial optimization problem $\Pi$, measures the relative position of the value of an approximated solution in the interval between the worst-value of $x$, i.e., the value of a worst feasible solution of $x$, and optimal-value of $x$, i.e., the value of a best solution of $x$.

Given a set of clauses (i.e., disjunctions) $C_{1}, \ldots, C_{m}$ on $n$ variables $x_{1}, \ldots, x_{n}$, MAX SAT (resp., MIN SAT) consists of determining a truth assignment to the variables that maximizes (minimizes) the number of clauses satisfied. On the other hand, given a set of cubes (i.e., conjunctions) $C_{1}, \ldots, C_{m}$ on $n$ variables $x_{1}, \ldots, x_{n}$, MAX DNF (resp., MIN DNF) consists of determining a truth assignment to the variables that maximizes (minimizes) the number of conjunctions satisfied. For an integer $k \geqslant 2$, MAX $k s A T, \max k$ DNF, MIN $k$ SAT, MIN $k$ DNF (resp., MAX E $k$ SAT, MAX E $k$ DNF, MIN E $k$ SAT, MIN E $k$ DNF) are the versions of MAX SAT, MAX DNF, MIN SAT, MIN DNF where each clause or conjunction has size at most (resp., exactly) $k$; we denote by $\mathrm{MAX} \mathrm{SAT}_{k}, \mathrm{MIN} \mathrm{SAT}_{k}, \mathrm{MAX} \mathrm{DNF}_{k}$ and $\mathrm{MIN} \mathrm{DNF}_{k}$, the problems variants where clauses or cubes have size at least $k$. Finally, let us quote two particular weighted satisfiability versions, namely, MAX wSAT and MIN WSAT. In the former, given a set of clauses $C_{1}, \ldots, C_{m}$ on $n$ variables $x_{1}, \ldots, x_{n}$, with non-negative integer weights $w(x)$ on any variable $x$, we wish to compute a truth assignment to the variables that both satisfies all the clauses and maximizes the sum of the weights of the variables set to 1 . We consider that the assignment setting all the variables to 0 (even if it does not satisfy all the clauses) is feasible and represents the worst-value solution for the problem. The latter problem is similar to the former one, up to the fact that we wish to minimize the sum of the weights of the variables set to 1 and that feasible is now considered the assignment setting all the variables to 1 .

A problem $\Pi$ in NPO is a quadruple $\left(\mathcal{I}_{\Pi}, \operatorname{Sol}_{\Pi}, m_{\Pi}, \operatorname{opt}(\Pi)\right)$ where:

- $\mathcal{I}_{\Pi}$ is the set of instances (and can be recognized in polynomial time); 
- given $x \in \mathcal{I}_{\Pi}, \operatorname{Sol}_{\Pi}(x)$ is the set of feasible solutions of $x$; the size of a feasible solution of $x$ is polynomial in the size $|x|$ of the instance; moreover, one can determine in polynomial time if a solution is feasible or not;

- given $x \in \mathcal{I}_{\Pi}$ and $y \in \operatorname{Sol}_{\Pi}(x), m_{\Pi}(x, y)$ denotes the value of the solution $y$ of the instance $x ; m_{\Pi}$ is called the objective function, and is computable in polynomial time; we suppose here that $m_{\Pi}(x, y) \in$ $\mathbb{N}$;

- $\operatorname{opt}(\Pi) \in\{\min , \max \}$.

Given an instance $x$ of an optimization problem $\Pi$ and a feasible solution $y \in \operatorname{Sol}_{\Pi}(x)$, we denote by $\operatorname{opt}_{\Pi}(x)$ the value of an optimal solution of $x$, and by $\omega_{\Pi}(x)$ the value of a worst solution of $x$. The standard approximation ratio of $y$ is defined as $r_{\Pi}(x, y)=m_{\Pi}(x, y) / \operatorname{opt}_{\Pi}(x)$, while the differential approximation ratio of $y$ is defined as $\delta_{\Pi}(x, y)=\left|m_{\Pi}(x, y)-\omega_{\Pi}(x)\right| /\left|\operatorname{opt}_{\Pi}(x)-\omega_{\Pi}(x)\right|$.

For a function $f$ of $|x|$, an algorithm is a standard $f$-approximation algorithm (resp., differential $f$ approximation algorithm) for a problem $\Pi$ if, for any instance $x$ of $\Pi$, it returns a solution $y$ such that $r(x, y) \leqslant f(|x|)$, if opt $(\Pi)=\min$, or $r(x, y) \geqslant f(|x|)$, if opt $(\Pi)=\max ($ resp., $\delta(x, y) \geqslant f(|x|)$ ).

With respect to the best approximation ratios known for them, NPO problems can be classified into approximability classes. The most notorious among them are the following:

APX or DAPX: the class of problems for which there exists a polynomial algorithm achieving standard or differential approximation ratio $f(|x|)$ where function $f$ is constant (it does not depend on any parameter of the instance);

PTAS or DPTAS: the class of problems admitting a polynomial time approximation schema; such a schema is a family of polynomial algorithms $\left.\left.A_{\varepsilon}, \varepsilon \in\right] 0,1\right]$, any of them guaranteeing approximation ratio $1-\varepsilon$ (under the differential approximation paradigm and under the standard one in the case where $\operatorname{opt}(\Pi)=\max$ ), or $1+\varepsilon$ (under the standard approximation paradigm in the case where opt $(\Pi)=$ $\min )$;

FPTAS and DFPTAS: the class of problems admitting a fully polynomial time approximation schema; such a schema is a polynomial time approximation schema $\left(\mathrm{A}_{\varepsilon}\right)_{\varepsilon \in] 0,1]}$, where the complexity of any $\mathrm{A}_{\varepsilon}$ is polynomial in both the size of the instance and in $1 / \varepsilon$.

We now define a kind of reduction, called affine reduction and denoted by AF, which, as we will see, is very natural in the differential approximation paradigm.

Definition 1. Let $\Pi$ and $\Pi^{\prime}$ be two NPO problems. Then, $\Pi$ AF-reduces to $\Pi^{\prime}\left(\Pi \leq_{A F} \Pi^{\prime}\right.$ ), if there exist two functions $f$ and $g$ such that:

1. for any $x \in \mathcal{I}_{\Pi}, f(x) \in \mathcal{I}_{\Pi}$;

2. for any $y \in \operatorname{Sol}_{\Pi^{\prime}}(x), g(x, y) \in \operatorname{Sol}_{\Pi}(x)$; moreover, $\operatorname{Sol}_{\Pi}(x)=g\left(x, \operatorname{Sol}_{\Pi^{\prime}}(f(x))\right)$;

3. for any $x \in \mathcal{I}_{\Pi}$, there exist $K \in \mathbb{R}$ and $k \in \mathbb{R}^{\star}\left(k>0\right.$ if opt $(\Pi)=\operatorname{opt}\left(\Pi^{\prime}\right), k<0$, otherwise) such that, for any $y \in \operatorname{Sol}_{\Pi^{\prime}}(f(x)), m_{\Pi^{\prime}}(f(x), y)=k m_{\Pi}(x, g(x, y))+K$.

If $\Pi \leq_{\mathrm{AF}} \Pi^{\prime}$ and $\Pi^{\prime} \leq_{\mathrm{AF}} \Pi$, then $\Pi$ and $\Pi^{\prime}$ are called affine equivalent. This equivalence will be denoted by $\Pi \equiv{ }_{\mathrm{AF}} \Pi^{\prime}$.

It is easy to see that differential approximation ratio is stable under affine reduction. Formally, if, for $\Pi, \Pi^{\prime} \in$ NPO, $\mathrm{R}=(f, g)$ is an AF-reduction from $\Pi$ to $\Pi^{\prime}$, then for any $x \in \mathcal{I}_{\Pi}$ and for any $y \in \operatorname{Sol}_{\Pi^{\prime}}(f(x))$, $\delta_{\Pi}(x, g(x, y))=\delta_{\Pi^{\prime}}(f(x), y)$. Indeed, by Condition 2 of Definition 1, worst and optimal solutions in $x$ and $f(x)$ coincide. Since the value of any feasible solution of $\Pi^{\prime}$ is an affine transformation of the same solution seen as a solution of $\Pi$, the differential ratios for $y$ and $g(x, y)$ coincide also. Hence, the following holds. 
Proposition 1. If $\Pi \equiv_{\mathrm{AF}} \Pi^{\prime}$, then, for any constant $r$, any $r$-differential approximation algorithm for one of them is an $r$-differential approximation algorithm for the other one.

Optimization satisfiability problems as MIN SAT and MAX SAT are of great interest from both theoretical and practical points of view. On the one hand, the satisfiability problem (SAT) is the first complete problem for NP and MAX SAT, MIN SAT have generalizations or restrictions that are the first problems proved complete for numerous approximation classes under various approximability preserving reductions $([4,19])$. For instance, MaX 3SAT is APX-complete under the AP-reduction and Max-SNP-complete under the Lreduction ([17]), MAX WSAT and MIN WSAT are NPO-complete under the AP-reduction ([8]), etc. In general, many optimal satisfiability problems have for the polynomial approximation theory the same status as SAT for NP-completeness theory. On the other hand, many problems in mathematical logic and in artificial intelligence can be expressed in terms of versions of SAT; constraints satisfaction is one such version. Also problems in database integrity constraints, query optimization, or in knowledge bases can be seen as optimization satisfiability problems. Finally, some approaches to inductive inference can be modelled as Max SAT problems $([13,14])$. The interested reader can be referred to [5] for a survey on standard approximability of optimization satisfiability problems.

Let us note that differential approximability of the problems dealt here, has already been studied in [6]. There, among other results, it was shown that MAX SAT and MIN DNF, as well as MIN SAT and MAX DNF are equivalent for the differential approximation, that all these problems are not solvable by polynomial time differential approximation schemata, unless $\mathbf{P}=\mathbf{N P}$, and, finally, that MIN SAT cannot be approximately solved within differential approximation ratio $1 / m^{1-\epsilon}$, for any $\epsilon>0$ (where $m$ is the number of the clauses in its instance), unless $\mathbf{N P}=\mathbf{c o - R P}$. Finally, let us mention here that both MAX wSAT and MIN wSAT belong to 0-DAPX, the class of the problems for which no algorithm can guarantee differential approximation ratio strictly greater than 0 , unless $\mathbf{P}=\mathbf{N P}([16])$. This class has been also introduced in [6].

\begin{tabular}{l||c|c}
\hline \hline & Approximation ratios & Inapproximability bounds \\
\hline \hline MAX SAT & $4.34 /(m+4.34)$ & $\notin$ DAPX \\
MAX E2sAT & $17.9 /(m+19.3)$ & $11 / 12$ \\
MAX 3SAT & $4.57 /(m+5.73)$ & $1 / 2$ \\
MAX E3SAT & $8 /(m+8)$ & $1 / 2$ \\
MAX E $k$ SAT & $2^{k} /\left(m+2^{k}\right)$ & $1 / p, p$ the largest prime such that $3(p-1) \leqslant k$ \\
MIN SAT & $2 /(m+2)$ & \\
MIN (E) $k$ SAT & $2^{k} /\left(\left(2^{k-1}-1\right) m+2^{k}\right)$ & $1 / p, p$ the largest prime such that $3(p-1) \leqslant k$ \\
MIN 2SAT & $4 /(m+4)$ & $11 / 12$ \\
\hline \hline
\end{tabular}

Table 1: Summary of the main results of the paper.

In this paper, we further study differential approximability of MAX SAT, MIN SAT, MIN DNF and MAX DNF, and give approximation results and inapproximability bounds for several versions of these problems. A summary of the main results obtained is presented in Table 1. As one can see from the second column of the first line of this table, MAX SAT is not approximable within a constant approximation ratio, unless $\mathbf{P}=\mathbf{N P}$. This result is very interesting since it indicates that Max-NP ([17]) is not included in DAPX. This is an important difference with the standard approximability classes landscape where Max-NP $\subset$ APX. Another assessment with respect to our results is that the gap between lower and upper approximation bounds for the problems dealt is still large. However, this paper undertakes a systematic study of satisfiability problems in the differential paradigm, it extends the results of [6] and shows that none of the most classical satisfiability problems is in 0-DAPX. This approximability class has been introduced in [6] and represents the worst 
possible configuration for differential approximation since it includes the problems for which no polynomial time approximation algorithm can guarantee differential ratio greater than 0 . Inclusion of the problems dealt here in 0-DAPX or not, was a major question we handled since [6].

\section{Affine reductions between optimal satisfiability problems}

Let us first note that there does not exist general technique in order to transfert approximation results from differential (resp., standard) paradigm to standard (resp., differential) one, except for the case of maximization problems and for transferts between differential and standard paradigms. Proposition 2 just below deals with this last case.

Proposition 2. If a maximization problem $\Pi$ can be solved within differential approximation ratio $\delta$, then it can be solved within standard approximation ratio $\delta$, also.

Proof. Consider any differential polynomial time approximation algorithm A guaranteeing differentialapproximation ratio $\delta$ for any instance $x$ of a maximization problem $\Pi$. Denote by $\mathrm{A}(x)$, a solution computed by A when running on $x$. Then,

$$
\frac{m(x, \mathrm{~A}(x))-\omega(x)}{\operatorname{opt}(x)-\omega(x)} \geqslant \delta \Longrightarrow m(x, \mathrm{~A}(x)) \geqslant \delta \operatorname{opt}(x)+(1-\delta) \omega(x) \stackrel{\omega(x) \geqslant 0}{\Longrightarrow} \frac{m(x, \mathrm{~A}(x))}{\operatorname{opt}(x)} \geqslant \delta
$$

and the claim of the proposition is proved.

Corollary 1. Any standard inapproximability bound for a maximization problem $\Pi$ is also a differential inapproximability bound for $\Pi$.

We give in this section some affine reductions and equivalencies between the problems dealt in the paper. These results will allow us to focus ourselves only in the study of MAX SAT, MIN SAT and their restrictions without studying explicitly MAX and MIN DNF. We first recall a result already proved in [6].

Proposition 3. ([G]) MAX SAT $\equiv_{A F}$ MIN DNF and MIN SAT $\equiv_{A F}$ MAX DNF.

The following proposition shows that one can affinely pass from MAX $\mathrm{E} k \mathrm{saT}$ to MAX $\mathrm{E}(k+1)$ sat. This, allows us to transfert inapproximability bounds from MAX E3SAT to MAX EksAT, for any $k \geqslant 4$.

Proposition 4. $\mathrm{MAX}$ e $k \mathrm{sat} \leq \mathrm{AF} \operatorname{MAX} \mathrm{E}(k+1)$ sat.

Proof. Consider an instance $\varphi$ of max e $k$ sat on $n$ variables $x_{1}, \ldots, x_{n}$ and $m$ clauses $C_{1}, \ldots, C_{m}$. Consider also a new variable $y$ and build formula $\varphi^{\prime}$, instance of $\operatorname{MAX} \mathrm{E}(k+1)$ sat as follows: for any clause $C_{i}=\left(\ell_{i_{1}}, \ldots, \ell_{i_{k}}\right)$ of $\varphi$, where, for $j=1, \ldots, k, \ell_{i_{j}}$ is a literal associated with $x_{i_{j}}, \varphi^{\prime}$ contains two new clauses $\left(\ell_{i_{1}}, \ldots, \ell_{i_{k}}, y\right)$ and $\left(\ell_{i_{1}}, \ldots, \ell_{i_{k}}, \bar{y}\right)$. Hence, $\varphi^{\prime}$ is the conjunction of $2 m$ clauses of size $k+1$ on $n+1$ variables. Assume any truth assignment $T$ on the variables of $\varphi$ and denote by $(T, 1)$ (resp., $(T, 0)$ ) the extension of $T$ on $\varphi^{\prime}$ by setting $y=1$ (resp., $y=0$ ). Then, it is easy to see that $m\left(\varphi^{\prime},(T, 1)\right)=m\left(\varphi^{\prime},(T, 0)\right)=m+m(\varphi, T)$.

In other words, reduction just described, associating to any assignment $T^{\prime}$ of $\varphi^{\prime}$ its restriction $T$ on variables $x_{1}, \ldots, x_{n}$ as assignment for $\varphi$, is affine and the proof of the proposition is complete.

We now show that, for $k$ fixed, problems $k$ sAT and $k \mathrm{DNF}$ are affine equivalent.

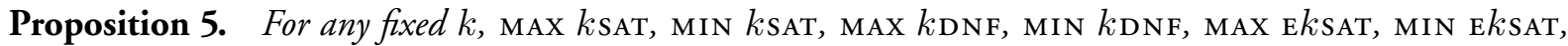
$\mathrm{MAX} \mathrm{E} k \mathrm{DNF}$ and MIN $\mathrm{E} k \mathrm{DNF}$ are all affine equivalent. 
Proof. We first prove affine equivalence between max $k$ sat and min $k$ sat. Given $n$ variables $x_{1}, \ldots, x_{n}$, denote by $\mathcal{C}_{k}$ the set of clauses of size $k$ and by $\mathcal{C}_{\leqslant k}$ the set of clauses of size at most $k$ on the set $\left\{x_{1}, \ldots, x_{n}\right\}$. Let us remark that any truth assignment verifies the same number $v_{k}$ of clauses on $\mathcal{C}_{k}$ and the same number $v_{\leqslant k}$ of clauses on $\mathcal{C}_{\leqslant k}$. Note also that, since $k$ is assumed fixed, sets $\mathcal{C}_{k}$ and $\mathcal{C}_{\leqslant k}$ are of polynomial size.

Let $\varphi$ be an instance of MAX E $k$ SAT on variable-set $\left\{x_{1}, \ldots, x_{n}\right\}$ and on a set $\mathcal{C}=\left\{C_{1}, \ldots, C_{m}\right\}$ of $m$ clauses. Consider instance $\varphi^{\prime}$ on the clause-set $\mathcal{C}^{\prime}=\mathcal{C}_{k} \backslash \mathcal{C}$. Then, for any truth assignment $T$ on $\left\{x_{1}, \ldots, x_{n}\right\}: m(\varphi, S)+m\left(\varphi^{\prime}, S\right)=v_{k}$; in other words, reduction just described is an affine reduction from MAX E $k$ SAT to MIN E $k$ SAT. Considering $\varphi$ as instance of MIN E $k$ SAT this time, the above describe an affine reduction from MIN $\mathrm{E} k \mathrm{SAT}$ to MAX $\mathrm{E} k \mathrm{SAT}$.

Furthermore, if $\mathcal{C}$ is an instance of MAX $k \mathrm{sAT}$, then we can see the clause-set $\mathcal{C}_{\leqslant k} \backslash \mathcal{C}$ as an instance of MIN $k$ SAT and the same arguments conclude an affine reduction from the former to the latter problem.

We now prove equivalence between versions of SAT and the corresponding versions of DNF. Given a clause $C=\left(\ell_{i_{1}} \vee \ldots \vee \ell_{i_{k}}\right)$ on $k$ literals, we build the cube (conjunction) $D=\left(\bar{\ell}_{i_{1}} \wedge \ldots \wedge \bar{\ell}_{i_{k}}\right)$. Any truth assignment $T$ on $\ell_{i_{j}}$ verifies $C$, if and only if it does not verify $D$, i.e., $m(C, T)=m-m(D, T)$. This specifies an affine reduction between MAX $\mathrm{E} k$ SAT and MIN E $k \mathrm{DNF}$, MIN E $k$ SAT and MAX E $k$ DNF, MAX $k$ SAT and MIN $k$ DNF and between MIN $k$ SAT and MAX $k$ DNF.

We finally show equivalence between MAX $k$ SAT and MAX E $k$ sAT. We first notice that the latter problem being a sub-problem of the former one, direction MAX E $k$ SAT $\leq \mathrm{AF}$ MAX $k$ SAT is immediate. On the other hand, as in Proposition 4, given an instance of MAX $k$ sAT, one can construct, for any clause of size at most $k$, a set of clauses of size exactly $k$, in such a way that this reduction is affine.

Combination of equivalencies shown above completes the proof of the proposition.

It is shown in [12], respectively (see also [4]), that MAX E3SAT is inapproximable within standard approximation ratio $(7 / 8)+\epsilon$, for any $\epsilon>0$, and MAX E2sAT is inapproximable within standard approximation ratio $(21 / 22)+\epsilon$, for any $\epsilon>0$ (in what follows for such results we will use, for simplicity, expression "within better than"). Discussion above, together with these bounds leads to the following result.

Proposition 6. max 2sat, max e2sat, min 2sat, min e2sat, max 2Dnf, maX e2dnf, min 2Dnf and MIN E2DNF are inapproximable within differential approximation ratio better than 21/22. Furthermore, for any $k \geqslant 3$, MAX $k \mathrm{SAT}$, MAX E $k$ SAT, MIN $k \mathrm{SAT}$ and MIN E $k \mathrm{SAT}$, MAX $k \mathrm{DNF}, \mathrm{MAX} \mathrm{E} k \mathrm{DNF}, \mathrm{MIN} k \mathrm{DNF}$ and MIN $\mathrm{E} k \mathrm{DNF}$, are inapproximable within differential approximation ratio better than $7 / 8$.

Proof. Concerning max 2sat and associates, Corollary 1 extends the result of [12] to the differential paradigm. Then, Proposition 5 suffices to conclude the proof.

For MAX $k$ sAT and associates, Corollary 1 extends the result of [15] to the differential paradigm, for MAX 3 sat and Proposition 5 transferts it to max e3sat. Then, Proposition 4 extends it for any $k \geqslant 4$. Finally, Proposition 5 suffices to conclude the proof.

Since the satisfiability problems stated in Proposition 6 are particular cases either of MAX SAT, or of MIN SAT, or of MAX DNF, or, finally, of MIN DNF, application of Proposition 6 and of Proposition 3 concludes the following corollary.

Corollary 2. MAX SAT, MIN SAT, MAX DNF and MIN DNF are inapproximable within differential approximation $7 / 8$.

Results of Corollary 2 are not the best ones. In Section 4, we strengthen the one for max sat. On the other hand, as it is proved in [6], MIN SAT is inapproximable within differential ratio better than $m^{\epsilon-1}$, for any $\epsilon>0$. Proposition 5 has to be used with some precautions in order to yield positive or negative approximation results. Indeed, if one of the problem stated in it is approximable within constant differential approximation ratio (i.e., within ratio that does not depend on an instance parameter), then this ratio is naturally transferred to all the other problems. A contrario, one can see in the proof of Proposition 5 that in many cases the number of the clauses for the derived instance can be much larger that the one for the initial 
instance. In such cases, if we deal with ratios functions of $m$ the form of these ratios is certainly preserved but not their value. For instance, assume that some problem $\Pi$ among the ones stated Proposition 5 is approximable within ratio $f(|\varphi|)$, where $|\varphi|$ denotes the number of clauses, or cubes, in $\varphi$, and $f$ decreases with $|\varphi|$. Assume also that there exists another problem $\Pi^{\prime}$ (among the ones stated in Proposition 5) such that $\Pi^{\prime} \leq_{\mathrm{AF}} \Pi$ and, furthermore, that this affine reduction transforms a formula $\varphi^{\prime}$ of $\Pi^{\prime}$ into a formula $\varphi$ for $\Pi$. Then, it transforms an approximation ratio $f(|\varphi|)$ for the latter into an approximation ratio $f\left(\left|\varphi^{\prime}\right|\right)$ for the former but, if the values $|\varphi|$ and $\left|\varphi^{\prime}\right|$ are very different the one from the other, then the values of the corresponding ratios do so.

In fact, one can easily observe that affine reductions of Proposition 5 perform the following differential ratio transformations:

- reduction from MAX E $k$ SAT to MIN E $k$ SAT transforms ratios $f(m, n)$ into $f\left((2 n)^{k}-m, n\right)$;

- reduction from MAX $k$ SAT to MIN $k$ SAT transforms ratios $f(m, n)$ into $f\left((2 n+1)^{k}-m, n\right)$;

- reductions between SAT and DNF are invariant for approximation ratios;

- reduction from max $k$ SAT to MAX E $k$ SAT transforms ratios $f(m, n)$ into $f\left(2^{k-1} m, n+k-1\right)$.

In other words, dealing with common approximability of the problems stated in Proposition 5, the following remarks hold:

- if one of these problems is in DAPX, then all the other ones are so;

- problems maX $k$ SAT,MAX E $k$ SAT, MIN $k$ DNF and MIN E $k$ DNF are approximable within differential ratios of $O(f(m))$ for a function $f$ strictly decreasing with $m$ if and only if one of them is $O(f(m))$ differentially approximable for $f(m)=O\left(m^{\alpha}\right)$, for some $\alpha>0$, or $f(m)=O(\log m)$; the same holds for the quadruple MIN $k$ SAT,MIN E $k$ SAT, MAX $k$ DNF and MAX E $k$ DNF;

- all problems are in Log-DAPX (the class of problems differentially approximable within ratios of $O(1 / \log |x|))$ if and only if one of them is so (observe that reductions dealt transform differential ratios of $O(\log m)$ into ratios of the form $O(\log m)$ or of $O(\log n)$, and ratios of $O(\log n)$ into ratios of the same form).

Finally, reduction of Proposition 4 transforms ratios $f(m, n)$ into $f(2 m, n+1)$.

\section{Positive results}

\subsection{Maximum satisfiability}

Consider an instance $\varphi$ of an optimal satisfiability problem, defined on $n$ variables $x_{1}, \ldots, x_{n}$ and $m$ clauses $C_{1}, \ldots, C_{m}$; consider also algorithm RSAT assigning at any variable value 1 with probability $1 / 2$ and, obviously, value 0 with probability $1 / 2$.Then, denoting by $\operatorname{Sol}(\varphi)$, the set of the $2^{n}$ possible truth assignments for $\varphi$, and by $E(\operatorname{RSAT}(\varphi))$ the expectation of a solution computed by $\operatorname{RSAT}$ when running on $\varphi$, the following holds: $E(\operatorname{RSAT}(\varphi))=\sum_{T \in \operatorname{Sol}(\varphi)} m(\varphi, T) / 2^{n}$.

Algorithm RSAT can be derandomized by the following technique denoted by DSAT. For $i=1, \ldots, n$ :

- compute $E_{i}^{\prime}=E\left(m(\varphi, T) \mid x_{i}=1\right)$ and $E_{i}^{\prime \prime}=E\left(m(\varphi, T) \mid x_{i}=0\right)$, where $T$ is a random assignment and the values of the $i-1$ first variables have already been fixed in iterations $1, \ldots i-1$;

- set $x_{i}=1$, if $E_{i}^{\prime} \geqslant E_{i}^{\prime \prime}$; otherwise, set $x_{i}=0$.

Lemma 1. $m(\varphi, \operatorname{DSAT}(\varphi)) \geqslant E(\operatorname{RSAT}(\varphi))$. 
Proof. It is easy to see that $E(\operatorname{RSAT}(\varphi))=\left(E_{1}^{\prime} / 2\right)+\left(E_{1}^{\prime \prime} / 2\right)$; hence $\max \left\{E_{1}^{\prime}, E_{1}^{\prime \prime}\right\} \geqslant E(\operatorname{RSAT}(\varphi))$. Furthermore, at any of the $n$ steps of DSAT, $\max \left\{E_{i}^{\prime}, E_{i}^{\prime \prime}\right\}=\left(E_{i+1}^{\prime} / 2\right)+\left(E_{i+1}^{\prime \prime} / 2\right) \leqslant \max \left\{E_{i+1}^{\prime}, E_{i+1}^{\prime \prime}\right\}$. We so have $E(\operatorname{RSAT}(\varphi)) \leqslant \max \left\{E_{1}^{\prime}, E_{1}^{\prime \prime}\right\} \leqslant \max \left\{E_{n}^{\prime}, E_{n}^{\prime \prime}\right\}=\operatorname{DSAT}(\varphi)$, that concludes the proof of the lemma.

Note finally, that DSAT is polynomial since, for any $i=1, \ldots, n$, computation of $E_{i}^{\prime}$ and $E_{i}^{\prime \prime}$ is performed in polynomial time. Indeed, for any such computation it suffices to determine with what probability any clause of $\varphi$ is satisfied and to sum these probabilities over all the clauses of $\varphi$.

We are ready now to state and prove positive differential approximation results for the problems dealt here.

Proposition 7. Algorithm DSAT achieves for MAX E $k$ SAT differential approximation ratio $2^{k} /\left(\operatorname{opt}(\varphi)+2^{k}\right)$. This ratio is bounded below by $2^{k} /\left(m+2^{k}\right)$.

Proof. Note first that we can assume that $\operatorname{opt}(\varphi)>\omega(\varphi)$ (otherwise, MAX E $k$ SAT would be polynomial on $\varphi$ ). Then,

$$
\omega(\varphi)<E(\operatorname{RSAT}(\varphi)) \leqslant m(\varphi, \operatorname{DSAT}(\varphi))
$$

From (1) and given that feasible values of $\mathrm{MAX} \mathrm{E} k \mathrm{SAT}$ are integer, we get:

$$
m(\varphi, \operatorname{DSAT}(\varphi))-\omega(\varphi) \geqslant 1
$$

Since clauses in $\varphi$ are of size $k$, the expectation that any of them is satisfied equals $1-2^{-k}$. Hence,

$$
m(\varphi, \operatorname{DSAT}(\varphi)) \geqslant E(\operatorname{RSAT}(\varphi))=m\left(1-\frac{1}{2^{k}}\right) \geqslant \operatorname{opt}(\varphi)\left(1-\frac{1}{2^{k}}\right)
$$

Using (2) and (3), we get:

$$
\delta(\varphi, \operatorname{DSAT}(\varphi)) \geqslant \max \left\{\frac{1}{\operatorname{opt}(\varphi)-\omega(\varphi)}, \frac{\operatorname{opt}(\varphi)\left(1-\frac{1}{2^{k}}\right)-\omega(\varphi)}{\operatorname{opt}(\varphi)-\omega(\varphi)}\right\}
$$

The first term in (4) is increasing with $\omega(\varphi)$, while the second one is decreasing. Equality holds when $\omega(\varphi)=\left(\operatorname{opt}(\varphi)\left(1-2^{-k}\right)\right)-1$. In this case, (4) gives

$$
\delta(\varphi, \operatorname{DSAT}(\varphi)) \geqslant \frac{2^{k}}{\operatorname{opt}(\varphi)+2^{k}} \geqslant \frac{2^{k}}{m+2^{k}}
$$

Last inequality in (5) holding thanks to the fact that $\operatorname{opt}(\varphi) \leqslant m$, qed.

Notice that the ratio claimed by Proposition 7 increases with $k$. This is quite natural since for $k>$ $\log m$, MAX $k$ SAT is polynomial. Indeed, using (3) with such a $k$, we get $m(\varphi, \operatorname{DSAT}(\varphi)) \geqslant \operatorname{opt}(\varphi)-$ $(\operatorname{opt}(\varphi) / m)>\operatorname{opt}(\varphi)-1$, i.e., $m(\varphi, \operatorname{DSAT}(\varphi))=m$, since the feasible values of MAX $k$ sat are integer.

We now propose a reduction transferring approximation results for MAX SAT problems from standard to differential paradigm. It will be used in order to achieve differential approximation results for MAX SAT, MAX 3SAT and MAX 2sAT.

Proposition 8. If a maximum satisfiability problem is approximable on an instance $\varphi$, within standard approximation ratio $\rho$, then it is approximable in $\varphi$ within differential approximation ratio $\rho /((1-\rho) \omega(\varphi)+1)$.

Proof. Fix any maximum satisfiability problem $\Pi$, sharing the ones dealt until now, and assume that there exists a polynomial time algorithm achieving standard approximation ratio $\rho$ for $\Pi$. Consider an instance $\varphi$ of $\Pi$, run both A and DSAT on $\varphi$ and retain assignment $T$ satisfying the maximum number of clauses 
between $\mathrm{A}(\varphi)$ and $\operatorname{DSAT}(\varphi)$. Obviously, $m(\varphi, T) \geqslant \rho \operatorname{opt}(\varphi)$. Hence, the differential approximation ratio of $T$ is

$$
\delta(\varphi, T) \geqslant \frac{m(\varphi, T)-\omega(\varphi)}{\frac{m(\varphi, T)}{\rho}-\omega(\varphi)}
$$

Since, as we have seen in the proof of Proposition 7, $m(\varphi, T) \geqslant \omega(\varphi)+1$, (6) becomes

$$
\delta(\varphi, T) \geqslant \frac{1}{\frac{\omega(\varphi)+1}{\rho}-\omega(\varphi)}=\frac{\rho}{(1-\rho) \omega(\varphi)+1}
$$

The proof of the proposition is now complete.

From the result of Proposition 8, we can deduce several corollaries by specifying values for $\omega(\varphi)$ and $\rho$. The main such corollaries are stated in the propositions that follow. Before stating and proving them, let us remark that, in the case of MAX $k$ SAT

$$
E(\operatorname{RSAT}(\varphi)) \leqslant m\left(1-\frac{1}{2^{k}}\right)
$$

Then (1) and (8) yield:

$$
\omega(\varphi) \leqslant m\left(1-\frac{1}{2^{k}}\right)
$$

Proposition 9. MAX SAT is approximable within differential approximation ratio $4.34 /(m+4.34)$.

Proof. We can assume $\omega(\varphi) \leqslant m-1$, otherwise $(\omega(\varphi)=m)$ all feasible solutions of $\varphi$ have the same value. Since $1-\rho \geqslant 0$, the differential ratio of (7) decreases with $\omega(I)$. So, it suffices to substitute $m-1$ for $\omega(\varphi)$, to use the fact that MAX SAT is approximable within standard ratio 1/1.2987 ([3]), and the proof of the proposition is complete.

Proposition 10. maX E2sat is approximable within differential approximation ratio $17.9 /(m+19.3)$, and MAX 3sat within $4.57 /(m+5.73)$.

Proof. For max 2sat, remark first that, using (3), the expectation of the solution computed by the random algorithm RSAT is, using (9), less than, or equal to, $3 \mathrm{~m} / 4$. Consequently, $\omega(\varphi) \leqslant 3 \mathrm{~m} / 4$. Next, the fact that MAX SAT is approximable within standard ratio 1/1.0741 ([10]) suffices to conclude the proof.

For $\operatorname{Max} 3 \mathrm{sat}, \omega(\varphi) \leqslant 7 m / 8$ and $\rho=1 / 1.249([18])$.

\subsection{Minimum satisfiability}

We finish this section by studying MIN SAT and some of its versions. Before stating our results, we note that algorithm RSAT can be derandomized in an exactly symmetric way, in order to provide a solution for MIN $k$ SAT with value smaller than expectation's value.

Proposition 11. If a minimum satisfiability problem is approximable on an instance $\varphi$, within standard approximation ratio $\rho$, then it is approximable in $\varphi$ within differential approximation ratio

$$
\frac{\rho}{(\rho-1)\left(1-\frac{1}{2^{k}}\right) m+\rho}
$$

Proof. As in the proof of Proposition 7, since we deal with a minimization problem, (1) becomes:

$$
\operatorname{opt}(\varphi) \leqslant m(\varphi, \operatorname{DSAT}(\varphi)) \leqslant E(\operatorname{RSAT}(\varphi))<\omega(\varphi)
$$

Consequently, (2) becomes:

$$
m(\varphi, \operatorname{DSAT}(\varphi))-\omega(\varphi) \leqslant 1
$$


Considering the best among the solutions computed by DSAT and A (the $\rho$-standard approximation algorithm assumed for MIN $k$ SAT in the statement of the theorem), denoting it by $T$ and using (10) and (11), we get:

$$
\delta(\varphi, T) \geqslant \max \left\{\frac{1}{\omega(\varphi)-\operatorname{opt}(\varphi)}, \frac{\omega(\varphi)-\rho \operatorname{opt}(\varphi)}{\omega(\varphi)-\operatorname{opt}(\varphi)}, \frac{\omega(\varphi)-m\left(1-\frac{1}{2^{k}}\right)}{\omega(\varphi)-\operatorname{opt}(\varphi)}\right\}
$$

where the third term in (12) is due to the fact that $T$ has a better value than the value of algorithm RSAT.

The first term in (12) is decreasing with $\omega(\varphi)$, while the second and third ones are increasing. We distinguish two cases depending on the relation between these terms.

If the second term is greater than the third one, i.e., if $\rho \operatorname{opt}(\varphi) \leqslant m\left(1-2^{-k}\right)$, then equality of the first two terms of (12) is achieved when $\omega(\varphi)=1+\rho \operatorname{opt}(\varphi)$. In this case, (12) gives:

$$
\delta(\varphi, T) \geqslant \frac{\rho}{(\rho-1) m\left(1-\frac{1}{2^{k}}\right)+\rho}
$$

If, on the other hand, second term is smaller than the third one, i.e., if $\rho \operatorname{opt}(\varphi) \geqslant m\left(1-2^{-k}\right)$, then equality of the first and the third term in (12) is achieved when $\omega(\varphi)=m\left(1-2^{-k}\right)+1$. In this case also, $\delta(\varphi, T)$ verifies (13). The proof of the proposition is now complete.

The best standard approximation ratios known for MIN $k$ SAT and MIN SAT are $2\left(1-2^{-k}\right)$ and 2, respectively ([7]). With the ratio just mentioned for MIN $k$ SAT, the result of Proposition 11 can be simplified as indicated in the following corollary.

Corollary 3. MIN $k$ SAT is approximable within differential ratio $2^{k} /\left(\left(2^{k-1}-1\right) m+2^{k}\right)$.

Proposition 12. MIN SAT is approximable within differential ratio $2 /(m+2)$.

Proof. Use Proposition 11 with $\rho=2$ ([7]).

Also, using Corollary 3 with $k=2$ and $k=3$, the following corollary holds and concludes the section.

Corollary 4. MIN 2SAT and MIN 3SAT are approximable within differential ratios $4 /(m+4)$ and $8 /(3 m+8)$, respectively.

\section{Inapproximability}

We first recall some basics about $\operatorname{MAX} \mathrm{E} 3 \operatorname{Lin} p$ that will be used for deriving our results. In this problem, we are given a positive prime $p, n$ variables $x_{1}, \ldots, x_{n}$ in $\mathbb{Z} / p \mathbb{Z}, m$ linear equations of type $\alpha_{i_{\ell}} x_{i_{\ell}}+\alpha_{j_{\ell}} x_{j_{\ell}}+$ $\alpha_{k_{\ell}} x_{k_{\ell}}=\beta_{\ell}$ and our objective is to determine an assignment on $x_{1}, \ldots, x_{n}$, in such a way that a maximum number among the $m$ equations is satisfied.

As it is proved in [12] (see also [9] for the case where all the coefficients equal 1), for any $p \geqslant 2$ and for any $\epsilon>0, \operatorname{MAX} \mathrm{E} 3 \operatorname{LIN} p$ cannot be approximated within standard approximation ratio $(1 / p)+\epsilon$, even if coefficients in the left-hand sides of the equations are all equal to 1 . Note that, due to Corollary 1 , this bound is immediately transferred to the differential paradigm.

Finally, let us quote the following GAP-reduction (see [2] for more about this kind of reductions), proved in [12], that will be used in order to yield our results.

Proposition 13. ([12]) Given a problem $\Pi \in N P$ and a real $\delta>0$, there exists a polynomial transformation $g$ from any instance $I$ of $\Pi$ into an instance of MAX E3LIN 2 such that:

- if $I$ is a yes-instance of $\Pi$ (we use here classical terminology from [11]), then opt $(g(I)) \geqslant(1-\delta) m$;

- if $I$ is a no-instance of $\Pi$, then $\operatorname{opt}(g(I)) \leqslant(1+\delta) m / 2$.

Proposition 13 shows, in fact, that MAX E3LIN 2 is not approximable within standard ratio $1 / 2+\epsilon$, for any $\epsilon>0$, because an algorithm achieving it would allow us to distinguish in polynomial time the yes-instances of any problem $\Pi \in \mathbf{N P}$ from the no-ones. Devising of such reductions is one of the most common strategies for proving inapproximability results in standard approximation. 


\subsection{Bounds for MAX E3SAT}

We first prove a GAP-reduction analogous to the one of Proposition 13 from any problem $\Pi \in \mathbf{N P}$ to maX E3sat. Note that this is the first time that a GAP-reduction is used in the differential approximation paradigm.

Proposition 14. Given a problem $\Pi \in N P$ and a real $\delta>0$, there exists a polynomial transformation $f$ from any instance I of $\Pi$ into an instance of MAX E3 sAT such that:

- if $I$ is a yes-instance of $\Pi$, then $\operatorname{opt}(f(I))-\omega(f(I)) \geqslant(1-2 \delta) m / 4$;

- if $I$ is a no-instance of $\Pi$, then $\operatorname{opt}(f(I))-\omega(f(I)) \leqslant \delta m / 4$

Proof. We first prove that the reduction of Proposition 13 can be translated into differential paradigm also. Consider an instance $I^{\prime}=g(I)$ of MAX E3LIN2 and a feasible solution $\vec{x}=\left(x_{1}, x_{2}, \ldots, x_{n}\right.$ ) for $I$ (we will use the same notation for both variables and their assignment) verifying $k$ among the $m$ equations of $I^{\prime}$. Then, vector $\vec{x}=\left(1-x_{1}, \ldots, 1-x_{n}\right)$, verifies the $m-k$ equations not verified by $\vec{x}$. In other words, $\operatorname{opt}(I)+\omega(I)=m$; hence, function $g$ claimed by Proposition 13 is such that:

- if $I$ is a yes-instance of $\Pi$, then $\operatorname{opt}\left(I^{\prime}\right)-\omega\left(I^{\prime}\right) \geqslant(1-2 \delta) m$;

- if $I$ is a no-instance of $\Pi$, then $\operatorname{opt}\left(I^{\prime}\right)-\omega\left(I^{\prime}\right) \leqslant \delta m$.

We are ready now to continue the proof of the proposition. Consider an instance $I$ of MAX E3LIN 2 on $n$ variables $x_{i}, i=1, \ldots, n$ and $m$ equations of type $x_{i}+x_{j}+x_{k}=\beta$ in $\mathbb{Z} / 2 \mathbb{Z}$, i.e., where variables and second members equal 0 , or 1 . In the same spirit as in [12], we transform $I$ into an instance $\varphi=h(I)$ of MAX E3SAT in the following way:

- for any equation $x_{i}+x_{j}+x_{k}=0$, we add in $h(I)$ the following four clauses: $\left(\bar{x}_{i} \vee x_{j} \vee x_{k}\right)$, $\left(x_{i} \vee \bar{x}_{j} \vee x_{k}\right),\left(x_{i} \vee x_{j} \vee \bar{x}_{k}\right)$ and $\left(\bar{x}_{i} \vee \bar{x}_{j} \vee \bar{x}_{k}\right)$

- for any equation $x_{i}+x_{j}+x_{k}=1$, we add in $h(I)$ the following four clauses: $\left(x_{i} \vee x_{j} \vee x_{k}\right)$, $\left(\bar{x}_{i} \vee \bar{x}_{j} \vee x_{k}\right),\left(\bar{x}_{i} \vee x_{j} \vee \bar{x}_{k}\right)$ and $\left(x_{i} \vee \bar{x}_{j} \vee \bar{x}_{k}\right)$.

It can immediately be seen that $h(I)$ has $n$ variables and $4 m$ (distinct) clauses.

Given a solution $y$ for MAX E3SAT on $h(I)$, we construct a solution $y^{\prime}$ for $I$ by setting $x_{i}=1$ if $x_{i}=1$ in $h(I)$ also; otherwise, we set $x_{i}=0$.

For instance, consider equation $x_{i}+x_{j}+x_{k}=0$ in $I$. It is verified if either 0 or 2 of the variables are equal to 1 . The several satisfaction possibilities for the clauses derived in $h(I)$ for this equation are the following:

- if zero, or two variables are set to 1 (true), then all the four clauses are satisfied;

- if one, or three variables are set to 1 , then 3 clauses are satisfied.

As a consequence, iterating this argument for any clause set built from an equation, we conclude that solution $y$ for maX E3 SAT on $h(I)$ verifies $m(h(I), y)=3 m+m\left(I, y^{\prime}\right)$. Since transformation between $y^{\prime}$ and $y$ is bijective, we get $\omega(h(I))=3 m+\omega(I)$ and opt $(h(I))=3 m+\operatorname{opt}(I)$. In other words, the reduction just described is an affine reduction from MAX E3LIN2 to MAX E3SAT.

It suffices now to remark that the composition $f=h \circ g$ verifies the statement of the proposition and its proof is concluded.

Proposition 14 has a very interesting corollary, expressed in the Proposition 15 just below, that exhibits another point of dissymmetry between standard and differential paradigms.

Proposition 15. Unless $\boldsymbol{P}=N \boldsymbol{P}$, no polynomial algorithm can compute, on an instance $\varphi$ of MAX E3SAT $a$ value that is a constant approximation of the quantity $\operatorname{opt}(\varphi)-\omega(\varphi)$. 
In view of Proposition 15, what is different between standard and differential paradigms with respect to the GAP-reduction is that in the former such a reduction immediately concludes the impossibility for a problem (assume that it is a maximization one) to be approximable within some ratio, by showing the impossibility for the optimal value to be approximated within this ratio. For that, it suffices that one reads the value of the solution returned by the approximation algorithm. In the latter paradigm such a conclusion is not always immediate. In fact, a reasoning similar to the one of the standard approximation is possible when computation of the worst solution can be done in polynomial time (this is, for instance, the case of maximum independent set and of many other NP-hard problems). In this case a simple reading of the value of the approximate solution is sufficient to give an approximation of opt $(x)-\omega(x)$. A contrario, when it is NP-hard to compute $\omega(x)$ (this is the case of the problems dealt here-simply think that the worst solution for MAX SAT is the optimal one for MIN SAT and that both of them are NP-hard -, of travelling salesman, etc.), then reading the value $m(x, y)$ of the approximate solution does not provide us with knowledge about $m(x, y)-\omega(x)$ and, consequently no approximation of opt $(x)-\omega(x)$ can be immediately estimated. So, use of GAP-reduction for achieving inapproximability results is different from the one paradigm to the other.

However, for the case we deal with, we will take advantage of a combination of Propositions 5 and 15 in order to achieve the inapproximability bound for MAX E3sat given in Proposition 16 that follows.

Proposition 16. Unless $\boldsymbol{P}=\boldsymbol{N P}$, MAX E3SAT is inapproximable within differential approximation ratio greater than $1 / 2$.

Proof. Assume that an approximation achieves differential ratio $\delta>1 / 2$, for max e3sat. Then, by Proposition 5, there exists an algorithm achieving the same differential ratio for MIN E3sat. Denote by $T_{1}$ and $T_{2}$, respectively, the solutions computed by these algorithms on an instance $\varphi$ of these problems. We have:

$$
m\left(\varphi, T_{1}\right)-\omega(\varphi) \geqslant \delta(\operatorname{opt}(\varphi)-\omega(\varphi))
$$

where opt $(\cdot)$ and $\omega(\cdot)$ are referred to MAX Е3sat. By the relations between all these parameters for the two problems specified in the proof of Proposition 5, we get:

$$
\operatorname{opt}(\varphi)-m\left(\varphi, T_{2}\right) \geqslant \delta(\operatorname{opt}(\varphi)-\omega(\varphi))
$$

Adding (14) and (15) member-by-member, we get $m\left(\varphi, T_{1}\right)-m\left(\varphi, T_{2}\right) \geqslant(2 \delta-1)(\operatorname{opt}(\varphi)-\omega(\varphi))$. So, simple reading of the values of $T_{1}$ and $T_{2}$, can provide us a constant approximation (since $\delta$ has been assumed to be a fixed constant greater than 1/2) of the quantity opt $(\varphi)-\omega(\varphi)$, impossible by Proposition 15 .

Proposition 16 together with Proposition 5 conclude the following corollary.

Corollary 5. For any $k \geqslant 3$, MAX $\mathrm{E} k \mathrm{SAT}$, MIN $\mathrm{E} k \mathrm{SAT}, \mathrm{MAX} k \mathrm{SAT}$ and $\mathrm{MIN} k \mathrm{SAT}$ are differentially inapproximable within ratios better than 1/2.

\subsection{MAX E $k$ SAT, $k \geqslant 3$}

In this section, we will generalize the GAP-reduction of Proposition 14 in order to further strengthen inapproximability results of Corollary 5.

Proposition 17. For any prime $p>0$, MAX e3LIn $p \leq \mathrm{AF}$ MAS E3( $p-1)$ SaT.

Proof. Consider a positive prime $p$ and an instance $I$ of $\operatorname{MAX} \operatorname{E} 3 \operatorname{Lin} p$ on $n$ variables and $m$ equations. Consider an equation $x_{1}+x_{2}+x_{3}=\beta$ (in $\mathbb{Z} / p \mathbb{Z}$ ) of $I$ and, for any $i=1,2,3, p-1$ new variables $x_{i}^{1}, \ldots, x_{i}^{p-1} \in\{0,1\}$. Consider, finally, equation

$$
\sum_{j=1}^{p-1} x_{1}^{j}+\sum_{j=1}^{p-1} x_{2}^{j}+\sum_{j=1}^{p-1} x_{3}^{j}=\beta
$$


It is easy to see that (16) is verified if and only if the number of variables set to 1 is either $\beta$ or $\beta+p$, or, finally, $\beta+2 p$.

Consider now the set of all the possible clauses on $3(p-1)$ literals issued from variables $x_{i}^{1}, \ldots, x_{i}^{p-1}$, $i=1,2,3$. Any truth assignment will satisfy all but one clause. For example, if any variable is assigned with 1 , the only unsatisfied clause is the one where all variables appear negative.

What is of interest for us is to specify when the number of variables set to 1 is either $\beta$ or $\beta+p$, or, $\beta+2 p$. For this, denote by $\mathcal{C}_{k}$ the set of clauses on $3(p-1)$ literals issued from variables $x_{i}^{1}, \ldots, x_{i}^{p-1}, i=1,2,3$ with exactly $k$ negative literals. Then, a truth assignment setting $k$ variables to 1 , verifies $\left|\mathcal{C}_{k}\right|-1$ clauses of $\mathcal{C}_{k}$, while any other truth assignment on the variables of $\mathcal{C}_{k}$ verifies all the $\left|\mathcal{C}_{k}\right|$ clauses. So, for an equation $x_{1}+x_{2}+x_{3}=\beta$, we will add in the instance of MAX E3 $(p-1)$ sat the set $\mathcal{C}_{k}$, for $k \in\{0, \ldots, 3(p-1)\}$ and $k \notin\{\beta, \beta+p, \beta+2 p\}$. Hence, if a truth assignment for these clauses has $\beta$, or $\beta+p$, or $\beta+2 p$ variables set to 1 , it will verify all the clauses constructed, otherwise it will verify all but one of these clauses.

In all, for any of the variables $x_{i}^{1}, \ldots, x_{i}^{p-1}$ we will build one new variable and we will transform any of the $m$ equations of $I$ into an equation as in (16). Then, for any of these new equations we add in the instance of MAX E3 $(p-1)$ SAT the set of clauses as built just above. The instance $\varphi$ of MAS E3 $(p-1)$ sat so constructed has $n(p-1)$ variables and, since the number of clauses issued from any equation is no more than $2^{3(p-1)}, \varphi$ will have at most $m_{\varphi} \leqslant m 2^{3(p-1)}$ clauses.

Given a truth assignment $T$ on the variables of $\varphi$, we set $x_{i}=\mid\left\{x_{i}^{k}: x_{i}^{k}=1\right.$ in $\left.T\right\} \mid$. Discussion above leads to $m(\varphi, T)=m_{\varphi}-m+m(I, S)$. On the other hand, it is easy to see that our reduction implies that any solution $S$ of $I$ is transformed into a truth assignment $T$ on the variables of $\varphi$ such that the relation between the values of $S$ and $T$ given just above is always satisfied. This relation confirms that the reduction specified is an affine one from MAX E3LIN $p$ to MAX E3 $(p-1)$ sat.

Finally, let us remark that it is possible that formula $\varphi$ contains many times the same clause. This, for instance, is the case if $I$ simultaneously contains equations say $x_{1}+x_{2}+x_{3}=\beta_{1}$ and $x_{1}+x_{2}+x_{3}=\beta_{2}$, for $\beta_{1} \neq \beta_{2}$. In this case, we can modify the construction described, by building the subset of $\mathcal{C}_{k}$ or $k \in\{0, \ldots, 3(p-1)\}$ and $k \notin\left\{\beta_{1}, \beta_{1}+p, \beta_{1}+2 p, \beta_{2}, \beta_{2}+p, \beta_{2}+2 p\right\}$. This concludes the proof of the proposition.

The result of Proposition 17 together with the result of [12] stated in the beginning of the section and Proposition 1, lead to the following corollary.

Corollary 6. For any prime $p$, MAX E3 $(p-1)$ sat is inapproximable within differential ratio greater than $1 / p$.

Furthermore, Propositions 4 and 5 allow us to rewrite Proposition 17 as follows.

Proposition 18. For any $k \geqslant 3$, neither $\mathrm{MAX} \mathrm{E} k \mathrm{SAT}$, nor MIN E $k \mathrm{SAT}$ can be approximately solved within differential ratio greater than $1 / p$, where $p$ is the largest positive prime such that $3(p-1) \leqslant k$.

Easy consequences of Proposition 18 are the following differential inapproximability bounds for several instantiations of maximum and minimum $k$-satisfiability:

- MAX and MIN 3SAT 4SAT and 5SAT are differentially inapproximable within ratio better than 1/2;

- MAX and MIN 6SAT, .., 11 sat are differentially inapproximable within ratio better than 1/3;

- MAX and Min 12sat, ., 17 sat are differentially inapproximable within ratio than $1 / 5, \ldots$

Finally, MAX SAT being harder to approximate than any MAX $k$ SAT problem, the following result holds and concludes the section.

Proposition 19. MAX SAT $\notin D A P X$. 
In [17] is defined a logical class of NPO maximization problems called MAX-NP. A maximization problem $\Pi \in$ NPO belongs to Max-NP if and only if there exist two finite structures $(U, \mathcal{I})$ and $(U, \mathcal{S})$, a quantifier-free first order formula $\varphi$ and two constants $k$ and $\ell$ such that, the optima of $\Pi$ can be logically expressed as:

$$
\max _{S \in \mathcal{S}}\left|\left\{x \in U^{k}: \exists y \in U^{\ell}, \varphi(\mathcal{I}, S, x, y)\right\}\right|
$$

The predicate-set $\mathcal{I}$ draws the set of instances of $\Pi$, set $\mathcal{S}$ the solutions on $\mathcal{I}$ and $\varphi$ the feasibility conditions for the solutions of $\Pi$. In the same article is proved that maX sat $\in$ Max-NP and that MAX-NP $\subset$ APX.

It is easy to see that (17) can be identically used in both standard and differential paradigms. So, Proposition 19 draws an important structural difference in the landscape of approximation classes in the two paradigms, since an immediate corollary of this proposition is that MAX-NP $\not \subset$ DAPX. We conjecture that the same holds for the other one of the celebrated logical classes of [17], the class MAX-SNP, i.e., we conjecture that MAX-SNP $\not \subset$ DAPX

\subsection{MAX E2SAT}

We have already seen in Proposition 6 that MAX E2sat is differentially inapproximable within ratio 21/22. In this section, we improve this result by operating an affine reduction from MAX E2LIN2 to MAX E2SAT.

Indeed, consider an instance $I$ of the former problem (on $n$ variables and $m$ equations) and an equation $x_{1}+x_{2}=0$ in $I$. Add in $\varphi$ (the instance of MAX E2sAT under construction) clauses $\bar{x}_{1} \vee x_{2}$ and $x_{1} \vee \bar{x}_{2}$. On the other hand, for an equation $x_{1}+x_{2}=1$, add in $\varphi$ clauses $x_{1} \vee x_{2}$ and $\bar{x}_{1} \vee \bar{x}_{2}$. Performing this transformation for any equation in $I$, we finally build a formula $\varphi$ of MAX E2saT on $n$ variables and $2 m$ clauses. Moreover, for any truth assignment $T$ on the variables of $\varphi$, one gets a solution $S$ for $I$ such that $m(\varphi, T)=m+m(I, S)$, qed.

It is shown in [12] that MAX E2LIN2 is inapproximable within standard approximation ratio better than 11/12. By Proposition 2, this bound is transferred to the differential paradigm. Then, the affine reduction just described concludes the following result.

Proposition 20. maX E2LIN2 $\leq_{\mathrm{AF}}$ MAX E2sat. Consequently, maX E2sat is differentially inapproximable within ratio greater than $11 / 12$.

\section{Ideas for further research}

We give in this concluding section a few ideas about possible ways for further improving results of the paper or for yielding new ones.

Consider a graph $G(V, E)$ of order $n$ and with maximum degree $\Delta$. We construct an instance $\varphi$ of MAX DNF on $n$ variable $x_{1}, \ldots, x_{n}$ and $n$ cubes $C_{1}, \ldots, C_{n}$ as follows: for any vertex $v_{i} \in V$, with neighbors $v_{i_{1}}, \ldots, v_{i_{\delta_{i}}}$, we add in $\varphi$ clause $x_{i} \wedge \bar{x}_{i_{1}} \wedge \ldots \wedge \bar{x}_{i_{\delta_{i}}}$. Let $T$ be a truth assignment satisfying $k$ cubes, say $C_{j_{1}}, \ldots, C_{j_{k}}$. Then, obviously, the vertex-set $V^{\prime}=\left\{v_{j_{1}}, \ldots, v_{j_{k}}\right\}$ is an independent set for $G$ (of size $k$ ). Conversely, given an independent set of $G$ of size $k$ consisting of vertices $v_{j_{1}}, \ldots, v_{j_{k}}$, the truth assignment setting variables $x_{j_{1}}, \ldots, x_{j_{k}}$ to 1 and any othe variable of $\varphi$ in 0 satisfies $k$ cubes. Observe finally that the size of the cubes built for $\varphi$ is bounded by $\Delta+1$. In all we have just exhibited an affine reduction from MAX INDEPENDENT SET- $\Delta$ (i.e., MAX INDEPENDENT SET on graphs with maximum degree bounded by $\Delta$ ) to $\operatorname{MAX} \Delta+1 \mathrm{DNF}$.

On the other hand, there exists an $\epsilon>0$ such that, for any $\Delta \geqslant 3$, MAX INDEPENDENT SET- $\Delta$ is not approximable within approximation ratio $1 / \Delta^{\epsilon}([1])$. Since standard and differential approximation ratios coincide for MAX INDEPENDENT SET (the worst independent set in a graph is the empty set), the result of [1] holds immediately for differential paradigm and can be used in order to conclude that there exists an $\epsilon>0$ such that, for any $k \geqslant 4, \operatorname{MAx} k \mathrm{DNF}$ is not differentially approximable within ratio greater than $1 / k^{\epsilon}$. This recovers the result of Proposition 19, namely, that MAX SAT $\notin$ DAPX. 
If one wishes to improve this result, a possible issue is the following. Recall that transformation of MAX $k$ DNF to MAX $k$ SAT of Proposition 5, consists of substituting any cube of size $\ell$ by $2 \ell-1$ clauses of size $\ell$. We so can affinely (but not polynomially) reduce MAX INDEPENDENT SET to MAX SAT by building an instance $\varphi$ of the latter on $n$ variabes and at most $n 2^{\Delta+1}$ clauses. But, if $\Delta$ is bounded by $\log n$, then this reduction is polynomial. In other words, if one obtains an inapproximability bound for MAX INDEPENDENT SET-log $n$ (for example a bound of the type $1 / \log ^{\epsilon} n$, for some positive $\epsilon$ ), then one can extend it immediately to MAX SAT improving so the bound of the paper.

\section{References}

[1] N. Alon, U. Feige, A. Wigderson, and D. Zuckerman. Derandomized graph products. Computational Complexity, 5:60-75, 1995.

[2] S. Arora and C. Lund. Hardness of approximation. In D. S. Hochbaum, editor, Approximation algorithms for NP-hard problems, chapter 10, pages 399-446. PWS, Boston, 1997.

[3] T. Asano, K. Hori, T. Ono, and T. Hirata. A theoretical framework of hybrid approaches to max sat. In Proc. International Symposium on Algorithms and Computation, ISAAC'97, volume 1350 of Lecture Notes in Computer Science, pages 153-162. Springer, 1997.

[4] G. Ausiello, P. Crescenzi, G. Gambosi, V. Kann, A. Marchetti-Spaccamela, and M. Protasi. Complexity and approximation. Combinatorial optimization problems and their approximability properties. Springer, Berlin, 1999.

[5] R. Battiti and M. Protasi. Algorithms and heuristics for max-sat. In D. Z. Du and P. M. Pardalos, editors, Handbook of Combinatorial Optimization, volume 1, pages 77-148. Kluwer Academic Publishers, 1998.

[6] C. Bazgan and V. Th Paschos. Differential approximation for optimal satisfiability and related problems. European J. Oper. Res., 147(2):397-404, 2003.

[7] D. Bertsimas, C-P. Teo, and R. Vohra. On dependent randomized rounding algorithms. In Proc. International Conference on Integer Programming and Combinatorial Optimization, IPCO, volume 1084 of Lecture Notes in Computer Science, pages 330-344. Springer-Verlag, 1996.

[8] P. Crescenzi, V. Kann, R. Silvestri, and L. Trevisan. Structure in approximation classes. SIAM J. Comput., 28(5):1759-1782, 1999.

[9] L. Engebretsen. Approximate Constraint Satisfaction. Phd thesis, Dept. of Numerical Analysis and Computing Science, Royal Institute of Technology, Stockholm, Sweden, 2000.

[10] U. Feige and M. X. Goemans. Approximating the value of two prover proof systems, with applications to max 2sat and max dicut. In Proc. Israel Symposium on Theory of Computing and Systems, ISTCS'95, pages 182-189, 1995.

[11] M. R. Garey and D. S. Johnson. Computers and intractability. A guide to the theory of NP-completeness. W. H. Freeman, San Francisco, 1979.

[12] J. Håstad. Some optimal inapproximability results. J. Assoc. Comput. Mach., 48:798-859, 2001.

[13] J. N. Hooker. Resolution vs. cutting plane solution of inference problems: some computational experience. Oper. Res. Lett., 7(1):1-7, 1988.

[14] A. P. Kamath, N. K. Karmarkar, K. G. ramakrishnan, and M. G. Resende. Computational experience with an interior point algorithm on the satisfiability problem. Ann. Oper. Res., 25:43-58, 1990. 
[15] H. Karloff and U. Zwick. A 7/8-approximation for max 3sat? In Proc. FOCS’97, pages 406-415, 1997.

[16] J. Monnot, V. Th. Paschos, and S. Toulouse. Approximation polynomiale des problèmes NP-difficiles : optima locaux et rapport différentiel. Informatique et Systèmes d'Information. Hermès, Paris, 2003.

[17] C. H. Papadimitriou and M. Yannakakis. Optimization, approximation and complexity classes. J. Comput. System Sci., 43:425-440, 1991.

[18] L. Trevisan, G. B. Sorkin, M. Sudan, and D. P. Williamson. Gadgets, approximation, and linear programming. In Proc. FOCS'96, pages 617-626, 1996.

[19] V. Vazirani. Approximation algorithms. Springer, Berlin, 2001. 